\title{
تأثير استيعاب المفردات العبية على مهارة التلاميذ في الكالام العربي لتلاميذالصف التاسع في مدرسة كاريسمى دار السلام المتوسطة الإسلامية المتكاملة كاراوانج
}

\section{Zakiyah $^{1}$ \\ Yan Septiana Prasetiadi ${ }^{2}$}

\section{ملخص البحث}

تأثير استيعاب المفردات العربية على مهارة التلاميذ في الكلام العربي. الأغراض هذا البحث لمعرفة استيعاب المفردات العربية وقلدة التلاميذ في الكالام العربي وأثر من استيعاب المفردات العربية على مهارة التلامين في الكلام العربي. إن المفردات هي

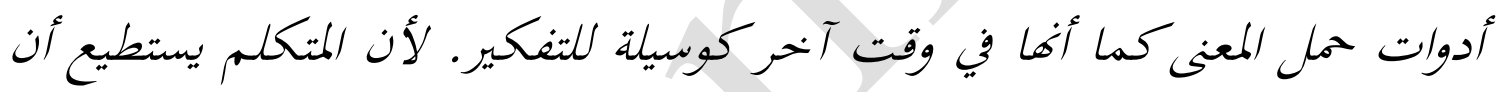
يفكر ثم يعبر ما خطر في باله وفكره بكلمات ما يريله. مهارة الكلام وسيلة مهمة

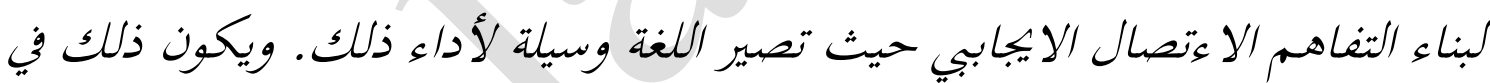
اللغة العربية انها وسيلة مهمة ايضا. وكان الكالام مهارة احد في اداء الاتصال باستعمال اللغة العربية كوسيلة له. هذا البحث هوالبحث الكمي يعنى بالمتغيرتين. ونوع هذا البحث هوالبحث الحالة. تستخلدم الباحثة المصادر المكتبية والميدانية لنيل البيانات في كتابة هذا البحث. وقل فعلت طريقة جمع البيانات بتوزيع الإستغتاء والإختبار ومواقبة الملاحظة وطريقة التوثيق. وقلد فعلت أملوب تحليل البيانات بالأداء العلمى microsoft excel لجمع البيانات أولا ثم استخدام برنامج (statistical package of social science) الأحصائي. إن حقيقة استيعاب المفردات العربية قد نضذت مع نشر ممتاز. لأن نتائج

${ }^{1}$ STAI DR. KH. EZ Muttaqien Purwakarta, zniswah@gmail.com.

${ }^{2}$ STAI DR. KH. EZ Muttaqien Purwakarta, yansprasetiadi@gmail.com. 


$$
\begin{aligned}
& \text { تحليل البيانات يشير إلى الحد الأدنى للقيمة التي تم الحصول عليها هوبر والقيمة }
\end{aligned}
$$

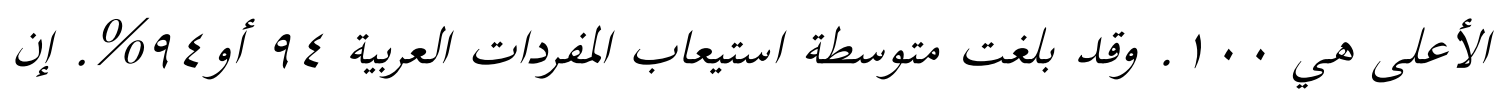

$$
\begin{aligned}
& \text { حقيقة مهارة التلاميذ في الكلام العربى قل نغذت مع نشر ممتاز لأن نتائج تحليل }
\end{aligned}
$$

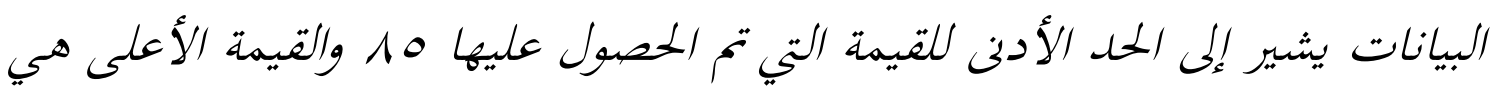

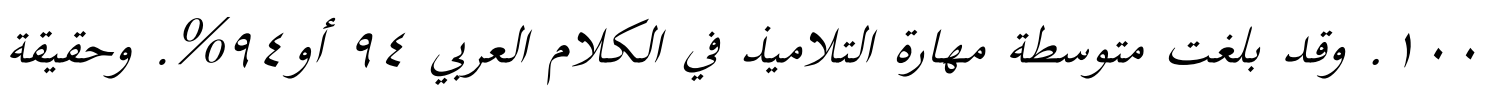

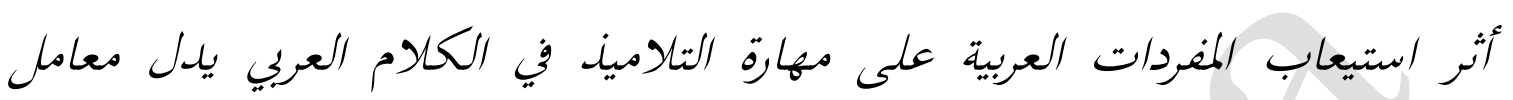

$$
\begin{aligned}
& \text { التحليد Rsquare البالغ ع • ع ، . هذا يلدل على الفهم أن المتغير الصادى يتأثر } \\
& \text { بالمتغير السينى على قدر · ؛ \%. والباقي يتأثر بعوامل أخري. } \\
& \text { الكلمات المفتاحية: المغردات، العربية، مهارة الكلام }
\end{aligned}
$$

\begin{abstract}
The effect of comprehension of Arabic vocabulary on students' skill in Arabic speaking. The purpose of this research is to know the comprehension of Arabic vocabulary and the students' ability in Arabic speech, and the effect of comprehension of Arabic vocabulary on students' skill in Arabic speech. Vocabulary is the means of carrying meaning as well as in another time as a means of thinking. Because the speaker can think and then express what came to his mind and thought in the words of what he wants. Speaking skill is an important tool for building understanding and positive communication, as language becomes a means to do so. And that in the Arabic language is an important way as well. Speech was someone's skill in performing communication using the Arabic language as his medium. This research is a quantitative research concerned with the two variables. The type of this research is case research. The researchers used both desk and field sources to obtain data in writing this paper. The method of data collection has done by distributing the questionnaire, testing, observation and documentation method. I have done the data analysis method using Microsoft Excel to collect the data first and then using the spss (statistical package of social science) program. for statistical calculation activities. The fact that the assimilation of Arabic vocabulary was carried out with excellent publication. Because the results of data analysis indicate that the minimum value obtained is 83 and the highest value is 100. The average Arabic vocabulary comprehension was 94 or $94 \%$. The fact that the students' skill in Arabic speaking was implemented with excellent publication because the results of data analysis indicate that the minimum value obtained is 85 and the highest value is 100. The average students' skill in Arabic speaking is 94 or 94\%. And the fact that the effect of
\end{abstract}

2 | Kalamuna: P-ISSN: 2655-4267, E-ISSN: 2745-6943 
comprehension of Arabic vocabulary on the students' skill in Arabic speaking indicates the Rsquare coefficient of 0.404. This indicates the understanding that the $y$ variable is affected by the sigmoid variable by an amount of $40 \%$. The rest is affected by other factors.

Keywords: Arabic, speaking skill, vocabulary.

مقلدمة

اللغة هي الة الاتصال ويستخدمها المعلم في عملية التعليم. باللغة يجعل

الناس على سهولة الاتصال مع الغير، يستطيعون على تبادل الافكار خاصة في التعليم.أن اللغة مهمة لنيل معرفة عن أي شيء كان. اللغة أيضا تنفع لوسيلة التعليم خصوصا في المدرسة الإسلامية أوالمعاهد العصرية.

اللغة هي أعظم الالآت التي يستخدمها الإنسان في تحقيق التعاون والاتصال بأبناء جنسه. وسبب ظهور لغة ما منطوقة كانت أم مكتوبة بدأ تاريخ الإنسان وبدأت معه الثقافة والحضارة، الأمر الذي يميز الإنسان، هذا الكائن المنغرد، عن غيره من خخلوقات الله.

اللغة العربية هي أكثر اللغات السامية تحدثا وإحدى أكثر اللغات انتشارا في العالم. اللغة العربية أيضا ذات أهمية قصوى لدى المسلمين، فهي عندهم لغة مقدسة إذا أها لغة القرآن، وهي لغة الصلاة وأساسية في القيام بالعديد من العبادات والشعائر الإسالمية. وتكون اللغة العربية يعني لغة رسمية في كل دول الوطن وأيضا في دولتنا الإندونسية.

3 Abdul Majid, 'كياهي إمام يحي محروس ودوره في تعليم اللغة العربية في معهد" المحروسية" ليربويو كديري: دراسة وصفية تحليلية (Universitas Islam Negeri Maulana Malik Ibrahim, 2013).

${ }^{4}$ Abdul Wahab Rosyidi and Mamlu'atul Ni'mah, 'Memahami Konsep Dasar Pembelajaran Bahasa Arab' (UINMaliki Press, 2011), p. 4. 
في الحقيقة أن تعليم اللغة العربية سهلا ولا صعبا بشرط أن يجتهد طالبها وأن يكون له شجاعة في استعمالها من غير خوف من الخطاء. وكذالك لابد ان يكثر التدريب على استخدامها.

مهارة الكالام وسيلة مهمة لبناء التفاهم الاءتصال الايجابي حيث تصير اللغة وسيلة لأداء ذلك. ويكون ذلك في اللغة العربية انها وسيلة مهمة ايضا. وكان الكام مهارة احد في اداء الاتصال باستعمال اللغة العربية كوسيلة له.

لا يمكن الناس يتكلمون بدون معرفة المفردات. المفردات هي قائمة الكلمة. \

المفردات صيغة جمع من المفردة هي بحموعة من المفردة، التي لديهم التعريف ووصف الترجمته ودون الربط من الكلمات الآخر وترتيب أبجاديا التي تستخدم من قبل شحص إما شفويا أوكتابيا.

كما عرفنا أن التلاميذ الذين لا يستطيعون التكلم باللغة العربية بسبب لم

يعرفوا المفردات. ولكن بعد التلاميذ يستطيعون على تكلم اللغة العربية قليلا فقليلا بسبب نقصان الإستيعاب المفردات. ولذالك معرفة المفردات والإستيعاب المفردات مهمة جدا. قد تعلم التلاميذ والتلميذات اللغة العربية مند صغارهم يعني من روضة الاطفال في المدرسة الإسلامية كاريسمى دار السلام. وقد يستخدمون اللغة العربية كل اليوم منذ السنة الخامسة حتى السنة التاسعة. ولكن بسسب وجود جائحة قورنا التلاميذ والتلميذات يتعلمون في بيتهم. وبهذا ينقصون على استخدام اللغة العربية. أن تعلم التلاميذ بسبب هذا الواقع بوسائل "zOOM" ولذالك نقصان التلاميذ علي اهتمام الدرس ما بين المدرس ويسبب على نقصان فهم التلاميذ عند

${ }^{5}$ Ahmad Fajar and Devi Kurniawati, 'Upaya Meningkatkan Hasil Belajar Siswa Dengan Media Flashcard Pada Materi An-Nazah Di DTA Manaarul Huda Kelas IV Ahmad Fajar 1 Devi Kurniawati 2', 2.1 (2021), 24-36.

${ }^{6}$ Ahmad Fuad Effendy, 'Metodologi Pengajaran Bahasa Arab', Malang: Misykat, 35 (2005), p. 110.

7 T KBBI, 'Kamus Besar Bahasa Indonesia Pusat Bahasa', Penerbit PT Gramedia Pustaka Utama, Jakarta, 2008, p. 527.

8 Zulhannan, Teknik Pembelajaran Bahasa Arab Interaktif (Jakarta: Rajawali Pers, 2014), p. 109.

4 | Kalamuna: P-ISSN: 2655-4267, E-ISSN: 2745-6943 
الدراسة. لا سيماعند المشكلة المتعددة يعني على صعوبة إشارة عند التعليم يسبب نقصان حضور التلاميذ عند الدراسة. وبهذا الحال ينقصون على التعامل مع الغير خصوصا على تكلم اللغة العربية. فلذالك تريد الباحثة أن تكتب البحث العلمى لهى بالموضوع " تأثير استيعاب المفردات العربية على مهارة التلاميذ في الكلام العربي". وتشخيص الباحثة موضوع هذا البحث يعني: نقصان التلاميذ على استيعاب المفردات، نقصان التلاميذ على استخدام اللغة العربية، نقصان التلاميذ على اهتمام عند الدرس، صعبة التلاميذ على فهم الدرس. مسائل البحث في هذا البحث "كيف

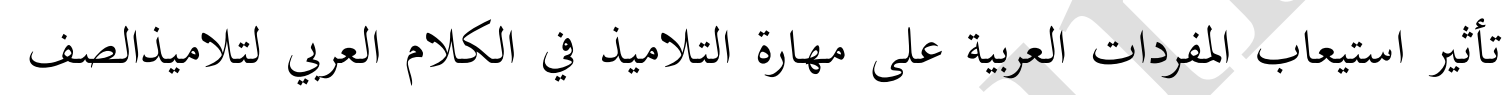

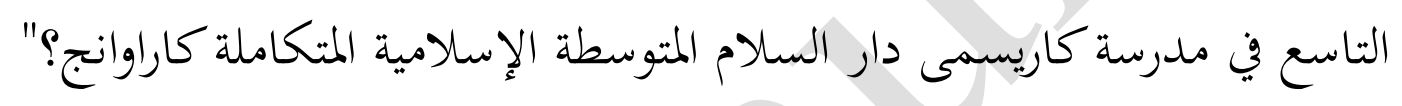
أهداف البحث في هذا البحث العلمى بمسائل البحث التالى: معرفة عن

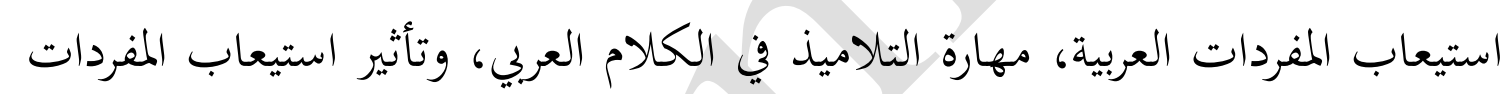
العربية على مهارة التلاميذ في الكام العربي، لتلاميذالصف التاسع في مدرسة كاريسمى دار السلام المتوسطة الإسلامية المتكاملة كاراوانج. فوائد البحث يعنى ترجوالباحثة ان تكون نتائج هذا البحث العلمى زيادة للخزانة في تربية اللغة وخاصة ما يتعلق بتعليم المفردات ومهارة الكلام، صارت نتائج هذا البحث العلمى مفيدة لمسؤولى تعليم اللغة العربية، صار هذا البحث العلمى خبرة للتلاميذ والباحثة خاصة وزيادة لخزانتهم العلمية.

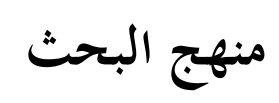

كان منهج البحث هوفي الأساس وسيلة علمية للحصول على البيانات لغرض وفائدة معينة.

${ }^{9}$ Sugiono, Metode Penelitian Pendidikan: (Pendekatan Kuantitatif, Kualitatif Dan R |\& D) (Alfabeta, 2008), p. 2. Kalamuna: P-ISSN: 2655-4267, E-ISSN: 2745-6943 | 5 
Kalamuna, Vol. 3. No. 1, Januari 2022. 01 - 14

إن تصميم هذا البحث العلمي هوالبحث الوصفى بالستعمال المدخل الكمي. والبحث باستعمال المدخل الكمي هوالبحث الذي بالستعمال استنتاجي. هذا المدخل اول من هيكل النظرية، فكرة العالم، وفهم الباحث على اساس كذا خبرته، ثم انتشر يصير مسائل وتحليل ليحصل صحيح في تأييد بيانات التجريبي. '1 المدخل الكمي بقصد يمتحن النظري، بناء الحقائق، يشير العلاقة بين المتغيرات، يعطي الوصف الإحصائى، يقدير النتائج. خطط البحث الذي باستعمال المخل الكمي هوواجب على تركيب، لفظة تدل على التفاعل، شكلي، ومهبأ بطيب قبله. خطط موصوف بالخصوص وبالتفسيل لأن خطط هوتخطيط البحث

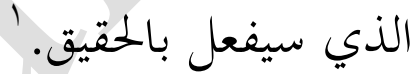

بحتمعية يتعلق بعناصر يعنى قسم مكان مأخوذ المعلومات، أما بوجود فرد واسرة، فرقة الاجتماع، مدرسة، فصل، منظمة وغير ذالكَ' ل. ومن ذالك تعريفات فيقال أن بحتمعة في هذا البحث هوجميع شخص أوجميع رعبة البحث يعنى جميع التلاميذ الصف التاسعة من المدرسة الثانوية الإسلامية المتكاملة كاريسمى دار السلام بكوت باروكاراوانج. وعدد جميع التلاميذ في هذا الفصل يعنى مب total تلميذات. فأخذت الباحثة جميع المحتمع عينة، فقامت الباحثة بالقبيل الكلي sampling الباحثة العينة من بحتمع التلميذات في الصف التاسع من المدرسة الثانوية الإسلامية المتكاملة كاريسمى دار السلام بكوت باروكاراوانج. للحصول على البيانات اللازمة في هذا البحث، يستخدم الباحثة عدة أنواع من أساليب جمع البيانات، كما يلي:

10 Ahmad Tanzeh, 'Metodologi Penelitian Praktis' (Yogyakarta: Teras, 2011), p. 99

11 Tanzeh, p. 10.

12 Nana Sudjana, 'Penelitian Dan Penilaian Dalam Pendidikan', Bandung: CV. Sinar Baru, 1989, p. 84.

6 | Kalamuna: P-ISSN: 2655-4267, E-ISSN: 2745-6943 
المقابلة هي طريقة لجمع البيانات للبحث عن المشاكل التي سبحت أوالبيانات لمكتسبة والمعلومات. وأما مقابلات التي عملت الباحثة في هذا البحث هي: مقابلة مع رئيس المدرسة، مقابلة مع مدرس اللغة العربية. ثم طريقة الاختبار هناك طريقتان لجمع البيانات في هذه الدراسة. يستخدم الاختبار الأول لجمع بيانات المفردات. والثاني هواختبار اللسان أوالشفوي لجمع قدرة التلاميذ

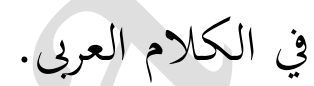
طريقة الوثيقية هي البخث عن البيانات حول الأمور أوالمتغيرات في شكل المذكرات، والنسخة، والكتب، والصحيفة، والبحلات، والنقوش، وليجير، وجدول الأعمال وغير

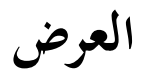 \\ استيعاب المفردات}

الاستيعاب لغة مصدر من "استوعب-يستوعب-استيعاب". وهوفهم

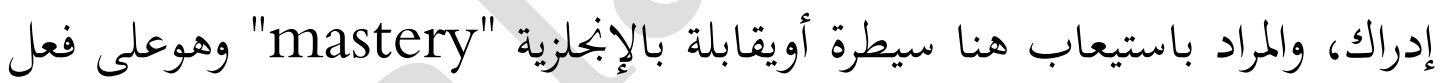
شيء أواستخدامه. وقال محمود أفندى، الاستيعاب هوالهدف النهائي لعملية القراءة. وهوعملية تكوين معان تنشأ من التكامل بين المعلومات التى يقدمها النص والمعلومات الكامنة في خلفية القرى المعرفة. أما في تعليم المفردات أن يتعلم الطلاب لنطق حروفها، بفهم معنها مستقلة، أومعرفة طريقة الاشتقاق منها. أوبحرد وصفها في تركيب لغوي صحيح. إن معيارالكفاءة في تعليم المفردات هوأن يكون الطلاب قادرا على هذا كله بالإضافة إلى اخر لا يقل عن هذا كله أهمية، وهوقدرته

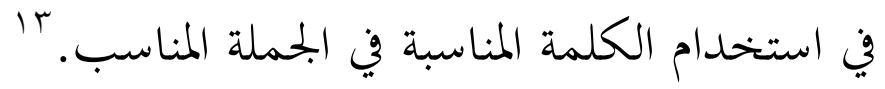


Kalamuna, Vol. 3. No. 1, Januari 2022. 01 - 14

المفردات واحدها مفردة. وهي اللغة أوالكلمة التى تتكون من حرفين فأكثر

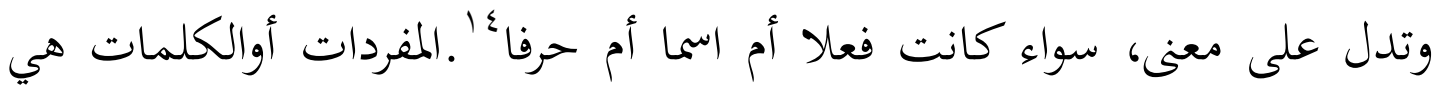
الواحدات التى تترتب أفقياء وفقا نظام نهوي خاص لتكوين الجملة. فهي بذالك أصغر وحدة لغوية حرة، وهي تختلف عن الصوت الذي هة أصغر وحدة لغوية ذات معنى أيظا، ولكنه قد يكون حرا أوغية حر.وهذا يعنى أن الكلمة قد تكون الكون صوتا واحدا أوأكثرا. - اك.

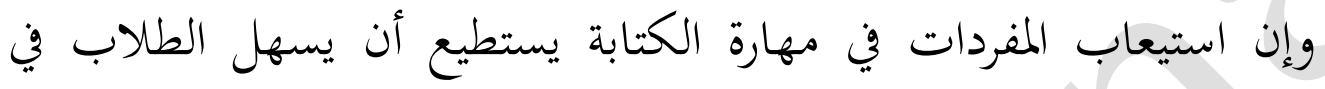
تعيين الكلمات، أهل تكون الكلمات مذكر أومؤنثا أوتكون إسما أوفعلا أوتكون بشكل المفرد أوالجمع أوغير ذلك.

نعتبر التلاميذ قادرة على استيعاب المفردات إدا وصلت بعض المؤشرات كما يلي: نطق المفردات وكتابتها جيدا وصحيحا، معرفة معان المفردات جيد، استخدام المفردات في جملة مفيدة شفويا كان أوكتابيا. طرق تدريس المفردات

إن تدريس المفردات يمكن بمراعات الأمور التالية، وهي (عحمد على الخولي): الاقترن المباشرة: ويجري هنا توضيح معنى الكلمة الجديد عن طريق اقتراهما بما يدل عليه مباشرة. ويستخدم هذا الأسلوب في توضيح معاقي الكلمات التى توجد

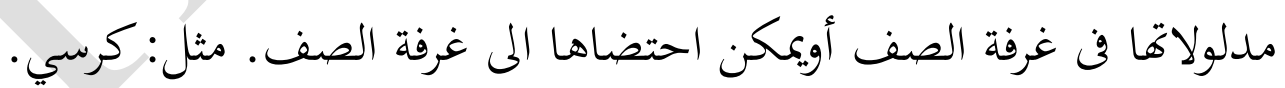
الصورية: إذا كان من غيرالممكن إحصار الشيء ذاته أوالمدلول عليه ذاته إلى غرفة الصف، فمن الممكن استخدم صورته لتوضيح معنى الكلمات. 


\section{تأثير استيعاب المفردات العربية... (Zakiyah \& Yan Septiana Prasetiadi)}

التمثيل: بعض الكلمات يستحسن توضيح معنها بالحركة وخحاصة إذاكان الكلمات أفعال، مثل: مشي، جلس، وقف، كتب ... الخ. السياق: من الممكن توضيح معنى الكلمات إذا وضعناها فن سياق لغوي يؤدي إلى كشف معنها. الترادف: في بعض الحالات، يتضح معنى الكلمات إذا ذكرنا كلمة ترادفها في المعنى بشرط أن تكون هذه الكلمة مألوفة لدى الطالب. التضاد: من الممكن توضيح معنى الكلمة بذكر كلمة مضادة لما ممائلة في الوظيفة النحوية بشرط أن تكون هذه الكلمة مألوفة أيضا لدى الطالب. التعريف: من الممكن شرح بعض الكلمات عن طريقة التعريف الترجمة: من الممكن شرح بعض الكلمات عن طريقة ترجمتها إلى اللغة الأم التى يتقنها الطالب، وخحاصة عند شرح الكلمات التى يصعب توضيح معانيها بالطرائق الأخرى.

\section{مفهوم مهارة الكالام}

المهارة من الكلمة مهرا - ومهورا - ومهارا ومهارة الشىء وفيه حذق فهوماهر. و ويقال مهر في العلم أى كان حاذقا عالما به. وتعريف المهارة بشكل عام بأها: أي شيء تعليم الفرد ليؤدية بسهولة ودقة. والمهارة بشكل عام هي السهولة والدقة في إجراء عمل منالأعمل، وهي تنمونتيجة لعملية التعليم. ومن معانى المهارة كما يذكر فؤاد أبوحطب، وصف الشخص بأنه على درجة من الكفاءة والجودة في الأداء. فالتركيز ينصب على مستوى الأداء وليس على خصائص الأداء ذاته، 
Kalamuna, Vol. 3. No. 1, Januari 2022. $01-14$

ومن ذلك مثلا مهارة النجار في استخدام المنشار، والمهارة في السباحة على الظهر

الكلام هولغة منطوقية الذي يعبره الإنسان عن الأحاسيس والمشاعر وخواطر الفرد. وما يريد أن يريد أن يجصله من معلومات وأفكار للأخرين بأسلوب سليم. ويمكن أيضا أن تفسر المهارات هي استعداد في سيء معين، أواستعداد الإكتساب شيء معين، وبالمثال يتضح المقال فمثلا المهارة لحم قطعتي حديد جزء من القدرة العامة في الحمادة الكلام من المهارة الأساسية التي يسعي الطالب إلى لى

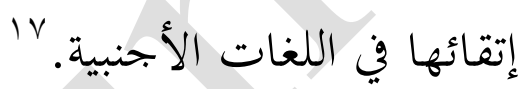
مهارة الكامام هي الكفاءة أوالملكة لتعبير الأصوات والألفاظ من حيث العقل مثل الفكرة والإرادة والشعور نحوى المتكلم به. وفي أوسع المعنى، الكام هونظام الخصائص المسموعة والمنظورة التي تستخدمها العضلات وأنسجة عضلية

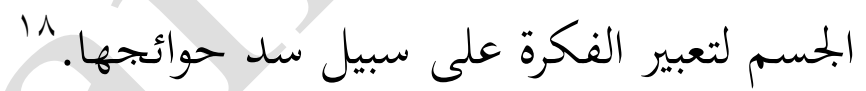
المستوى المتوسط: نطق الكلمات المنونة نطقا صحيحا يميز التنوين عن غيره من الظواهر، الاستجابة للأسئلة التي توجه إليه استجابة صحيحة مناسبة الهدف من إلقاء السؤال، إعادة سرد قصة تلقى عليه.

\section{أهداف تعليم مهارة الكلام}

أوضح محمود كامل الناقة ورشدي أحمد طعيمة' أن هناك أهداف عامة لتعليم الكلام يمكن أن نعرض لأهمها فيما يلي:

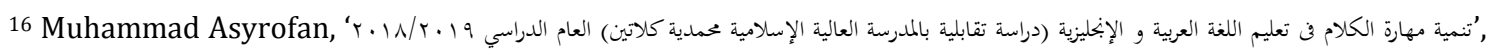
EL-HAYAH, 12.1 (2022).

17 Saiful Mustofa, 'تأثير استخدام وسيلة التعليم" لعبة دمية الأصابع" لترقية مهارة الكلام', Arabia, 9.2 (2019).

18 Acep Hermawan and Chaedar Alwasilah, Metodologi Pembelajaran Bahasa Arab (PT Remaja Rosdakarya, 2011), p. 135. 


\section{تأثير استيعاب المفردات العربية... (Zakiyah \& Yan Septiana Prasetiadi)}

( ) أن ينطق المعلم أصوات اللغة العربية وأن يؤدي أنواع التنغيم المختلفة وذلك

$$
\text { بطريقة مقبولة من أبناء العربية. }
$$

r) أن ينطق الأصوات المتجاورة والمتشابهة

r) أن يدرك الفرق في النطق بين الحركات القصيرة والحركات الطويلة

§) أن يعبر عن افكار مستخدما الصيغ النحوية المناسبة.

0) أن يعبر عن افكاره مستخدما النظم الصحيح لتركيب الكلمة في العربية خاصة في لغة الكاملام.

T) أن يستخدم بعض خصائص اللغة في التعبير الشفوي مثل التذكير والتأنيث وتمييز العلد والحال ونظام الفعل وأزمنته وغير ذلك مما يلزم المتكلم

( أن يكتسب ثروة لفظية كلامية مناسبة لعمره ومستوى نضجه وقدراته، وأن يستخدم هذه الثروة في إتمام عمليات اتصال عصرية.

م) أن يستخدم بعض أشكال الثقافة العربية المقبولة المناسبة لعمره ومستواه الاجتماعي زطبيعة عمله، وأن يكتسب بعض العلومات الأساسية عن

$$
\text { التراث العربي والاسلامي. }
$$

9) أن يعبر عن نفسه تعبيرا واضحا ومفهوما في مواقف الحلديث البسيطة. • (1) أن يتكن من التفكير باللغة العربية والتحدث بها بشكل متصل ومترابط

$$
\text { لفترات زمنية مقبولية. }
$$

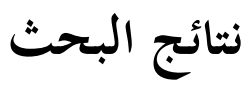

وقد أجريت التحليل بمساعدة البرنامج spss مع مستوى الدلالة هم (0 ., • ). تظهر نتائج اختبار تحليل المتطلبات المسبقة أن كلا البيانات لها بيانات 
Kalamuna, Vol. 3. No. 1, Januari 2022. 01 - 14

التوزيع العادي والخطي. نتيجة تحليل الارتباط باستخدام correlation pearson، القيمة التي تم الحصول عليها هبا, · يعني وجدت علاقة بين المتغير السينى والمتغير الصادى. الارتباط الإيجابي يعني أنه عندما تزداد جودة استيعاب المفردات، ستكون هناك زيادة في مهارة التلاميذ في الكلام العربي، والعكس بالعكس عندما تكون جودة استيعاب المفردات منخفضة، ثم تكون مهارة التلاميذ في الكلام العربي منخفضة أيضا.

واستنادا إلى نتائج اختبار الفرضية باستخدام قيمة ر الحساب مع رالكشف، معروف

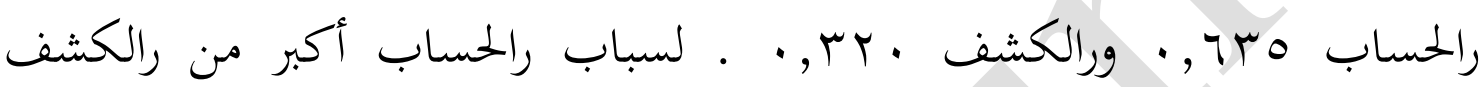
فالحاصلها وجدات علاقة مستوى بالدلالة بين المتغير السينى والمتغير الصادى. والقيمة الأهمية ا . .6، . يعني أقل من القيمة ه . . . فتدل هذه النتيجة على أن الفرضية الصفرية مردود بديل الفرضية مقبولة بمعتى أن بين المتغيرين أثر. وتأثير استيعاب المفردات العربية علي مهارة التلاميذ في الكلام العربي على قدر ·ــ والباقي يتأثر بعوامل أخري.

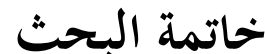

واستنادا إلى صياغة المشاكل، وأهداف البحث، والافتراضات البحثية، يمكن

أن تأخذ البيانات المتعلقة بنتائج البحث والمناقشة على سبيل الاستنتاج: إن حقيقة استيعاب المفردات العربية لدى التلاميذ فصل التاسع من المدرسة الثانوية الإسلامية المتكاملة كاريسمى دار السالام بكوت باروكاراوانج قد نفذت مع نشر ممتاز. لأن نتائج تحليل البيانات يشير إلى الحد الأدنى للقيمة التي تم الحصول عليها هو بم والقيمة الأعلى هي . . 1. وقد بلغت متوسطة استيعاب المفردات العربية §q أو ؟و\%. إن حقيقة مهارة التلاميذ في الكام العربى لدى التلاميذ فصل 
التاسع من المدرسة الثانوية الإسلامية المتكاملة كاريسمى دار السلام بكوت باروكاراوانج قد نفذت مع نشر ممتاز لأن نتائج تحليل البيانات يشير إلى الحد الأدنى للقيمة التي تم الحصول عليها مب والقيمة الأعلى هي . . 1 . وقد بلغت متوسطة مهارة التلاميذ في الكلام العربي عج أو و9\%. وحقيقة أثر استيعاب المفردات العربية على مهارة التلاميذ في الكلام العربي من المدرسة الثانوية الإسلامية المتكاملة كاريسمى دار السلام بكوت باروكاراوانج يدل معامل التحديد Rsquare البالغ ع ·ـ، • هذا يدل على الفهم أن المتغير الصادى يتأثر بالمتغير السينى على قدر • ع٪. والباقي يتأثر بعوامل أخري. وبصدر على امتحان الفرضية بمقارنة قيمة

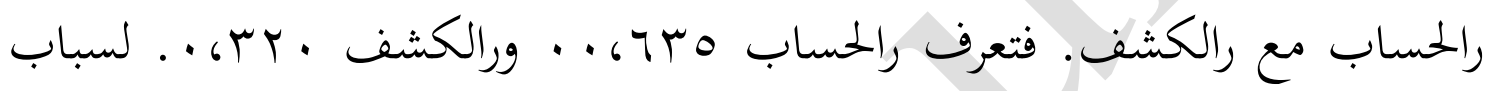
رالحساب أكبر من رالكشف فالحاصلها وجدت تأثير مستوى بالدلالة بين المتغير

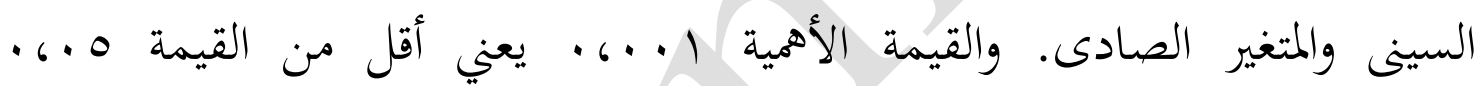
فالفرضية الصفرية مردودة الفرضية مقبولة

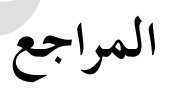

Asyrofan, Muhammad, 'تنمية مهارة الكلام فف تعليم اللغة العربية و الإنجليزية (دراسة تقابلية بالمدرسة العالية الإسلامية محمدية

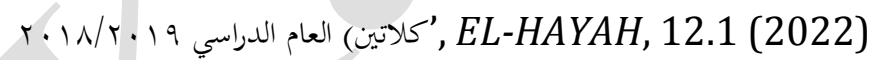

Effendy, Ahmad Fuad, 'Metodologi Pengajaran Bahasa Arab', Malang: Misykat, 35 (2005)

Fajar, Ahmad, and Devi Kurniawati, 'Upaya Meningkatkan Hasil Belajar Siswa Dengan Media Flashcard Pada Materi An-Nazah Di DTA Manaarul Huda Kelas IV Ahmad Fajar 1 Devi Kurniawati 2', 2.1 (2021), 24-36

Hermawan, Acep, and Chaedar Alwasilah, Metodologi Pembelajaran Bahasa Arab (PT Remaja Rosdakarya, 2011)

Irfan, Irfan, 'العلاقة بين حفظ القرآن واستيعاب المفردات في تعليم اللغة العربية بععهد العزة الإسلامي العالمي' (Universitas Islam Negeri Maulana Malik Ibrahim, 2020)

KBBI, T, 'Kamus Besar Bahasa Indonesia Pusat Bahasa', Penerbit PT Gramedia Pustaka Utama, Jakarta, 2008

Majid, Abdul, 'كياهي إمام يحي محروس ودوره في تعليم اللغة العربية في معهد" الخروسية" ليربويو كديري: دراسة وصفية تحليلية

(Universitas Islam Negeri Maulana Malik Ibrahim, 2013) 
Kalamuna, Vol. 3. No. 1, Januari 2022. 01 - 14

Mustofa, Saiful, 'تأثير استخدام وسيلة التعليم" لعبة دمية الأصابع" لترقية مهارة الكام', Arabia, 9.2 (2019)

Rosyidi, Abdul Wahab, and Mamlu'atul Ni'mah, 'Memahami Konsep Dasar Pembelajaran Bahasa Arab' (UIN-Maliki Press, 2011)

Sudjana, Nana, 'Penelitian Dan Penilaian Dalam Pendidikan', Bandung: CV. Sinar Baru, 1989

Sugiono, Metode Penelitian Pendidikan: (Pendekatan Kuantitatif, Kualitatif Dan $R \mid \&$ D) (Alfabeta, 2008)

Tanzeh, Ahmad, 'Metodologi Penelitian Praktis' (Yogyakarta: Teras, 2011)

Zulhannan, Teknik Pembelajaran Bahasa Arab Interaktif (Jakarta: Rajawali Pers, 2014)

عبدالمنعم حسن الملك, 'اسس اعداد الكتب التعليمية لغير الناطقين بالعربية', ب . . and رالغالى, ناصر عبدالله

طعيمة, رشدي أحمد, 'تعليم اللغة العربية لغير الناطقين بما مناهجه وأساليبه. الرباط: إسيكو ', 1919 